\title{
Study on the Prediction Method of Fusion Recognition for Characteristic Information of Coal Spontaneous Combustion
}

\author{
Wei-Feng Wang, Jun Deng, Yuan-Bin Hou, and Nai-Guo Wang
}

\begin{abstract}
To solve the technical problems of identification for the dangerous degree of coal spontaneous combustion, the coal spontaneous combustion process is divided into three stages: slow oxidation, accelerating oxidation and intense oxidation in the paper. The prediction method of fusion identification for characteristic information of coal spontaneous combustion is proposed and the sensitivity index of coal spontaneous combustion degree is determined. The quantitative relationship of the characteristic temperature of coal spontaneous combustion and the gas concentration of each index is determined by polynomial least squares fitting method according to the sample test data. The different feature states are classified by SVM and PSO-SVM algorithm. The criterion of the diagnosis and early warning of coal spontaneous combustion is given according to the results of the data level and feature level. The proposed method can effectively solve the problem of low recognition rate. The experiment shows that the prediction classification accuracy of SVM is $80 \%$, the prediction classification accuracy of PSO - SVM is approximately $100 \%$. The PSO-SVM algorithm can significantly improve the prediction accuracy compared with the traditional method, which provides criterion for the diagnosis and early warning of coal spontaneous combustion. The classification identification of the dangerous degree of coal spontaneous combustion is implemented. It is of great significance and practical application value for improving the level of prevention and control technology of coal spontaneous combustion early hazards.
\end{abstract}

Index Terms-Coal spontaneous combustion, characteristic information, fusion recognition, prediction method.

\section{INTRODUCTION}

In recent years, with the continuous development of the shallow coal resources and mine mining strength increase, the scope of goaf is larger and larger, and generates a lot of air leakage passage, mining activity and atmospheric pressure are changed, goaf is dynamic. It is easy to appear

Manuscript received November 17 2016; revised March 28, 2017. This work was supported in part by the financial support National Natural Science Foundation Funded Project (51504186, 51574193), Industrial Technology Research of Shaanxi Province Government (2016GY-191), Scientific Research Special of Education Department of Shaanxi Province Government (14JK1477).

Wei-Feng Wang and Jun Deng are with the School of Safety Science and Engineering, Xi' an University of Science and Technology, Xi'an, Shaanxi 710054, China (corresponding author: Jun DENG; tel.: +86-29-85583749; e-mail:251044098@qq.com,693167478@qq.com).

Yuan-Bin Hou is with the School of Electrical and Control Engineering, Xi'an University of Science and Technology, Xi'an, Shaanxi 710054, China (e-mail: 739053026 @qq.com).

Nai-Guo Wang is with the Shandong Xinjulong Energy Limited Liability Company, Heze, Shandong 274918, China (e-mail: 81864920@qq.com). "breathing" phenomenon, and then causes the coal spontaneous combustion [1], [2]. The data resources "dig" degree of the existing monitoring system is not enough, it's a lack of information in the danger degree of coal spontaneous combustion and the decision-making of prevention and control. The evolution environment of the coal spontaneous combustion is complicated, the liquidity of gas is poor, early hidden danger is difficult to identify in a timely manner. Poor heat conductivity properties, high temperature point hidden, single index, the degree of coal spontaneous combustion is judged not easily. The scope of coal fire area is very broad, artificial inspection workload is larger, blind area is more, non-response rates is higher. The internal connection of index gases and the temperature is not clear, the criterion of danger degree diagnosis and early warning is lack. The pertinence of the existing fire prevention and control means is not strong, the conventional measures do not have efficiency and boast skillful [3]. These problems have brought a lot of confusion for prevention and control work of goaf coal spontaneous combustion. For a long time, many scholars at home and abroad has carried on the basic theory research and scientific experiments to conquer these difficulties [4]-[7]. Due to the complexity of the problem, many influence factors, the limitation of the detection means, although some progress has been made, but has not been satisfactory solution. Therefore, the research of the prediction method of the fusion recognition for the characteristic information of coal spontaneous combustion is of great significance to the improvement of early coal spontaneous combustion hazard prevention and control technology.

The prediction method of the fusion recognition for the characteristic information of coal spontaneous combustion refers to coal in low temperature oxidation stage, external characterization information is not clear and not obvious signs of coal spontaneous combustion. Based on the characteristics of the coal oxidation heat release, the danger degree of coal spontaneous combustion is the anticipation in advance by fusion recognition algorithm. Therefore, it is necessary for the fusion recognition of the characteristic information of coal spontaneous combustion, the threat estimation and trend prediction. The fusion results are as a criterion of the diagnosis and early warning of coal spontaneous combustion, it will reduce the rate of false positives and non-response rates.

\section{INFORMATION FUSION ALGORITHM}

Information fusion algorithm main application fields 
include: automatic flight control, automatic target recognition, medical diagnosis, remote sensing, image processing, pattern recognition, robot, etc. [8]-[10]. Comprehensive literature both at home and abroad, multi-sensor information fusion technology has a big advantage. Each sensor information in time and space has certain complementary and redundancy. The time, space and frequency range of the system have been expanded. The blind area of single sensor is inevitable. The information collection and update rates were increased. All in all, multi-sensor information fusion technology is through a certain algorithm "merge" the information from multiple sources. Reliable and accurate information of sensors is produced more than single sensor information. And according to the information, reliable decision is made. Common fusion algorithm can be summarized as two categories: classical methods and modern methods. Two types of information fusion algorithm are shown in Table I [11]-[13].

TABLE I: THE ADVANTAGES AND DISADVANTAGES OF TwO KINDS OF INFORMATION FUSION ALGORITHMS

\begin{tabular}{|c|c|c|c|c|}
\hline & class & Main Algorithm & Advantages & Disadvantages \\
\hline \multirow{6}{*}{$\begin{array}{c}\text { Classical Fusion } \\
\text { Algorithm }\end{array}$} & \multirow{3}{*}{ Statistical Methods } & Bayesian Estimation Method & It has the axiomatic & \multirow[b]{2}{*}{$\begin{array}{l}\text { It needs more prior knowledge, the } \\
\text { applicable conditions are harsh. }\end{array}$} \\
\hline & & $\begin{array}{l}\text { D - S Evidence Theory } \\
\text { Method }\end{array}$ & $\begin{array}{l}\text { foundation, intuitive, easy to } \\
\text { understand, a small amount } \\
\text { of calculation. }\end{array}$ & \\
\hline & & Maximum Likelihood & \multirow{4}{*}{$\begin{array}{l}\text { Information loss is little, it is } \\
\text { suitable for the raw data } \\
\text { fusion. }\end{array}$} & \multirow{4}{*}{$\begin{array}{l}\text { It needs to get the accurate } \\
\text { mathematical model, for the } \\
\text { occasion of complex and difficult } \\
\text { to model cannot apply. }\end{array}$} \\
\hline & \multirow{3}{*}{ Estimation Methods } & Estimation & & \\
\hline & & Kalman Filter & & \\
\hline & & Least Square Method & & \\
\hline \multirow{4}{*}{$\begin{array}{l}\text { Modern Fusion } \\
\text { Algorithm }\end{array}$} & $\begin{array}{l}\text { Information Theory } \\
\text { Methods }\end{array}$ & Clustering Analysis & The priori knowledge of & \multirow{4}{*}{$\begin{array}{l}\text { Computational cost is larger, the } \\
\text { rules are difficult to establish or } \\
\text { learning time is long, It is not easy } \\
\text { to implement. }\end{array}$} \\
\hline & \multirow{3}{*}{$\begin{array}{l}\text { Artificial Intelligence } \\
\text { Method }\end{array}$} & Fuzzy Logic & $\begin{array}{l}\text { request, it has strong adaptive } \\
\text { capacity, it is easy to }\end{array}$ & \\
\hline & & Neural Network & $\begin{array}{c}\text { implement information } \\
\text { fusion between the subjective } \\
\text { and objective. }\end{array}$ & \\
\hline & & $\begin{array}{c}\text { Support Vector Machine } \\
\text { (SVM) }\end{array}$ & & \\
\hline
\end{tabular}

\section{The SENSITIVITy INDICATORS OF QuANTITATIVE RECOGNITION FOR THE DEGREE OF COAL SPONTANEOUS COMBUSTION}

In the experiment process of coal spontaneous combustion, each indicator gas can mutate at a certain temperature, its scope and characterization has certain regularity. According to the development change rule of gas concentration of $\mathrm{O}_{2}$, $\mathrm{N}_{2}, \mathrm{CH}_{4}, \mathrm{CO}, \mathrm{CO}_{2}, \mathrm{C}_{2} \mathrm{H}_{2}, \mathrm{C}_{2} \mathrm{H}_{4}, \mathrm{C}_{2} \mathrm{H}_{6}$ and their ratio along with the coal temperature, coal spontaneous combustion process is divided into three stages: slow oxidation, accelerating oxidation and intense oxidation. Each stage has a corresponding characterization parameters and a corresponding temperature range. The sensitivity indicators of quantitative recognition for the degree of coal spontaneous combustion are shown in Table II.

TABLE II: SENSITIVITY INDICATORS OF QUANTITATIVE RECOGNITION FOR COAL SPONTANEOUS COMBUSTION DEGREE

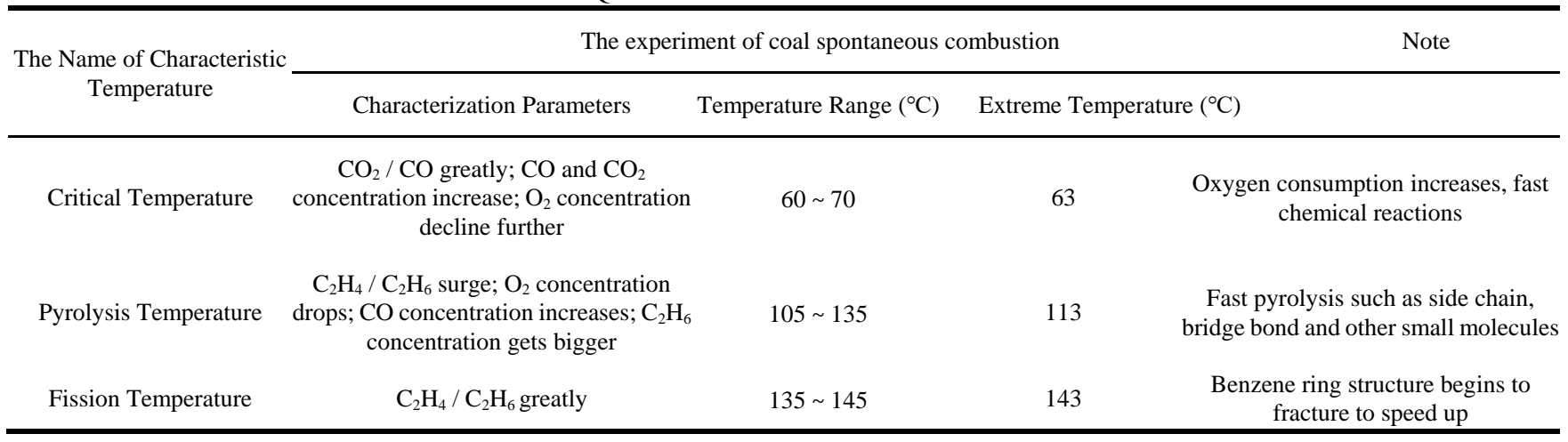

\section{THE ANTICIPATION MECHANiSM OF FUSION RECOGNITION FOR THE CHARACTERISTIC INFORMATION OF COAL SPONTANEOUS COMBUSTION}

The anticipation mechanism is to identify the current risk degree of coal spontaneous combustion and prevent the happening of the coal spontaneous combustion by the gas concentration and temperature of coal spontaneous combustion. The prediction method of fusion recognition for the characteristic information of coal spontaneous combustion was put forward based on the experiment data of coal spontaneous combustion and the support vector machine (SVM) algorithm.

Data level adopts the method of the polynomial least squares fitting, the correlation of coal temperature and indexes are established, and the corresponding relational 
expression is fitted. The quantitative relationship of the characteristic temperature of coal spontaneous combustion and the gas concentration of each index was determined according to the correlation expression between index gas and coal temperature based on least squares fitting.

PSO-SVM (Particle Swarm Optimization - support vector machine) fusion recognition prediction model of feature level of is established [14], [15]. PSO is used to analyze the SVM parameters $\mathrm{C}$ and $\gamma$ search optimization, SVM and kernel function parameters optimization is implemented, the sample data characteristic information of coal spontaneous combustion are used to train SVM. The change rule and trend prediction is implemented.

According to the results of the data level and feature level, the tendency and interconnectedness between coal spontaneous combustion index gases and temperature is determined. The diagnosis and early warning criterion of coal spontaneous combustion is given, the dangerous degree classification anticipation of coal spontaneous combustion is implemented.

\section{The Prediction Method of the Fusion ReCognition FOR THE CHARACTERISTIC INFORMATION OF COAL SPONTANEOUS COMBUSTION}

\section{A. Data Level Fusion Recognition}

The polynomial of each index gas parameters about temperature is fitted out by the least square method, Equation (1) is showed as follows:

$$
T_{j}=\sum_{i=0}^{n} a_{i} * Q_{j}^{i}, j=1,2, \ldots, 6
$$

Among them, $T$ represents temperature, $Q_{j}$ represents waiting for fitting gas concentration, $a_{i}, i=0,1 \ldots, n$ is need fitting parameters.

The relation between the temperature and the gas concentration is fitted respectively, temperature and fitting result of the gas concentration of $\mathrm{CO}, \mathrm{CH}_{4}$ is shown in Fig.1, Fig. 2. The upper part of each figure is the comparison of the fitting values and the original value between temperature and gas concentration. The lower part of each figure is the comparison of the initial temperature and the fitting temperature using gas concentration.
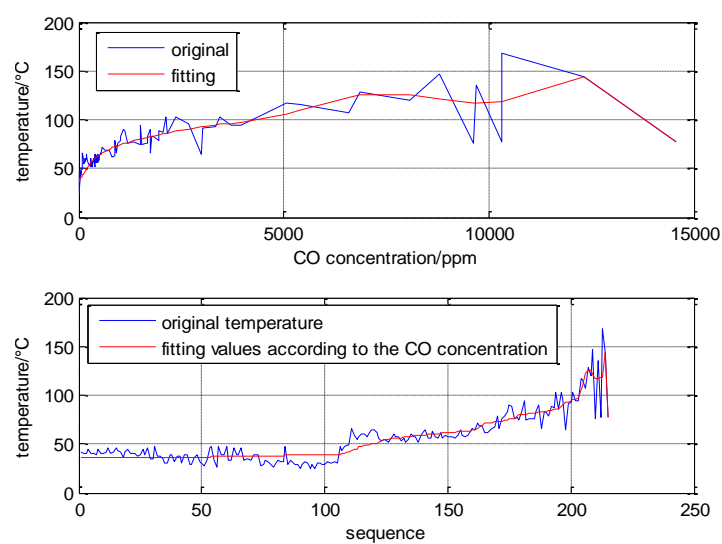

Fig. 1. The fitting relationship of temperature about $\mathrm{CO}$ concentration.
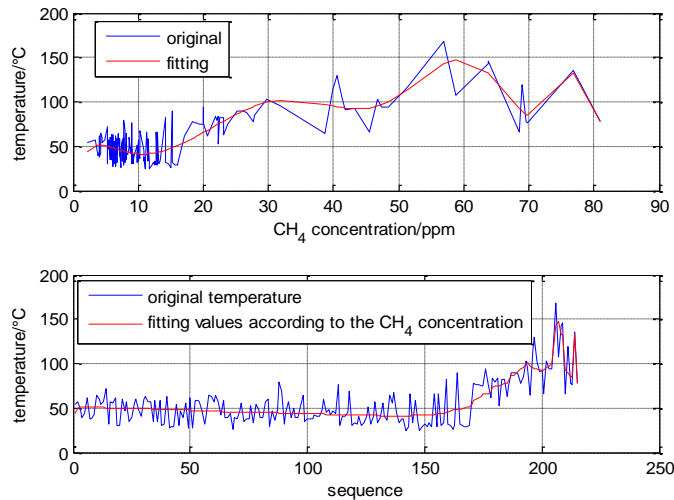

Fig. 2. The fitting relationship of temperature about $\mathrm{CH} 4$ concentration

Fig. 1 and Fig. 2 shows the fitting relationship of the temperature about the gas concentration of $\mathrm{CO}$ and $\mathrm{CH}_{4}$, if given the gas concentration, multiple fitting relationship about temperature is derived. Temperature fitting value about the gas concentration is the weighted average, and then gets mean value. According to the fitting expression of temperature of the gas concentration, and then fitting once more with the method of least squares, the express is showed as Eqs. (2):

$$
T=b_{0}+\sum_{j=1}^{n} b_{j} * T_{j}, n=6
$$

Among them, $\mathrm{T}$ represents integrated temperature fitting values, ${ }^{T_{j}}$ represents fitting temperature values about each index gas concentration, $b_{j}, j=0,1 \ldots, 6$ is need fitting parameters.

Temperature of each gas concentration is quadratic fitting, the result is shown in Fig. 3. It can be seen that the result of the quadratic fitting by the weighted average is closer to the test values of temperature, least squares method is used to implement data level fusion and the effect is better.

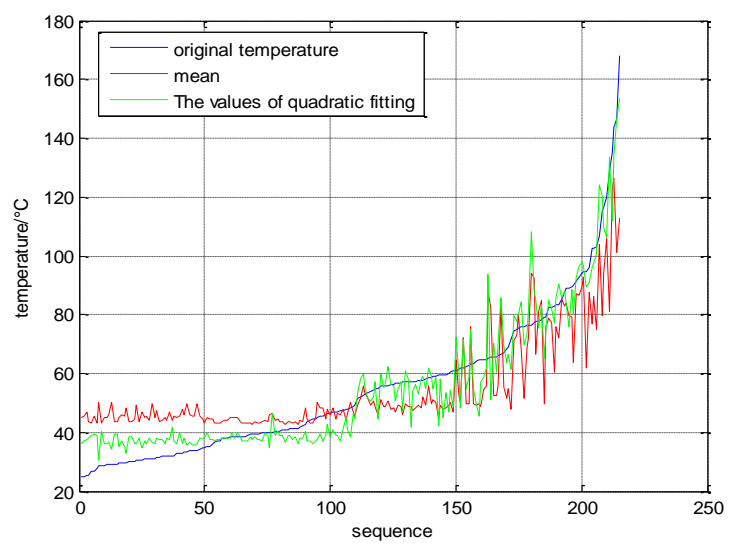

Fig. 3. Temperature about the fitting mean of the gas parameters and quadratic fitting values.

\section{B. Feature Level Fusion Recognition}

By the experiment platform of XK - II coal spontaneous combustion of Xi' an University of Science and Technology, coal spontaneous combustion characteristics of coal samples of a mine were studied. $\mathrm{O}_{2}, \mathrm{~N}_{2}, \mathrm{CO}, \mathrm{CO}_{2}, \mathrm{C}_{2} \mathrm{H}_{4}, \mathrm{C}_{2} \mathrm{H}_{2}, \mathrm{CH}_{4}$ and $\mathrm{C}_{2} \mathrm{H}_{6}$ gas concentration was used as input information of feature level.

Level of coal spontaneous combustion is divided into three 
stages: critical temperature $\sim$ pyrolysis temperature, pyrolysis temperature $\sim$ fission temperature, $>$ fission temperature.

The scope of the corresponding characteristic temperature are: $60 \sim 70{ }^{\circ} \mathrm{C}, 105 \sim 70{ }^{\circ} \mathrm{C}$ and $135 \sim 145^{\circ} \mathrm{C}$. Risk grade as output, the original data is divided into three categories, grade 1, 2, 3, respectively. Among them, 215 groups of data is regarded as the training sample, 30 groups of data is regarded as test samples.
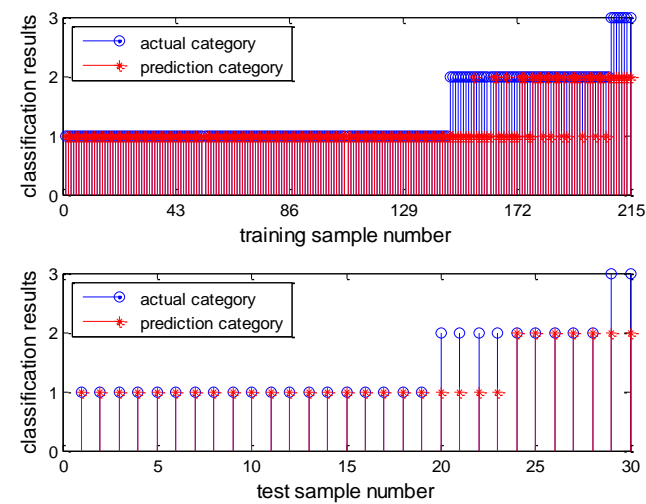

Fig. 4. The training and prediction results of SVM classification model.

In the matlab environment, SVM and PSO - SVM algorithm program is written by Libsvm toolbox, and then classified recognition, training and test results is shown as in Fig.4, Fig.5. It can see that the SVM model in Fig.4, the forecast accuracy of training samples is $81.86 \%(176 / 215)$, the prediction accuracy of test samples is $80 \%$ (24/30), test samples have six group forecast classification error.

It can see that the SVM model in Fig.5, the forecast accuracy of PSO - SVM model is 95.35\% (205/215), the forecast accuracy of test sample is approximate $100 \%(30 / 30)$ Comparing the SVM prediction results, the prediction model of PSO - SVM can significantly improve prediction accuracy.
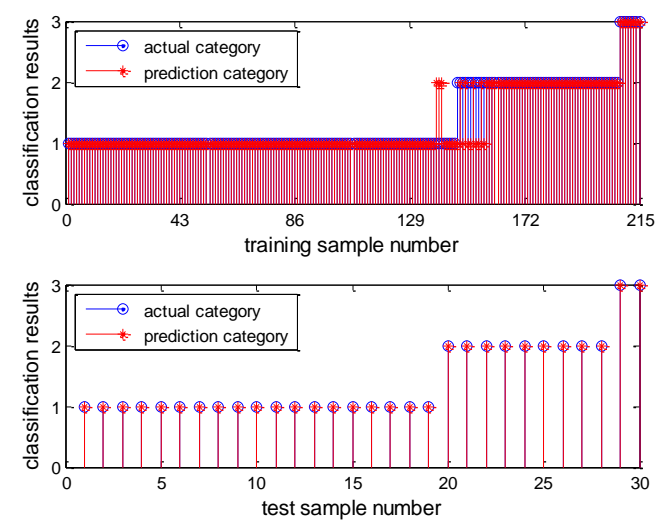

Fig. 5. The training and prediction results of PSO - SVM classification model.

By the same token, the PSO - SVM regression prediction model is established, and the mean square error of cross-validation is selected as fitness function value. The training and testing results of the regression prediction model of SVM and PSO - SVM are shown as in Fig.6, Fig.7, respectively. It can be seen that the difference of training prediction results and the actual value is very large in Fig.6. The prediction results and the actual value are basically identical in Fig. 7. In other words, PSO - SVM model can significantly improve the prediction accuracy, it provides more accurate and reliable information for policy makers.
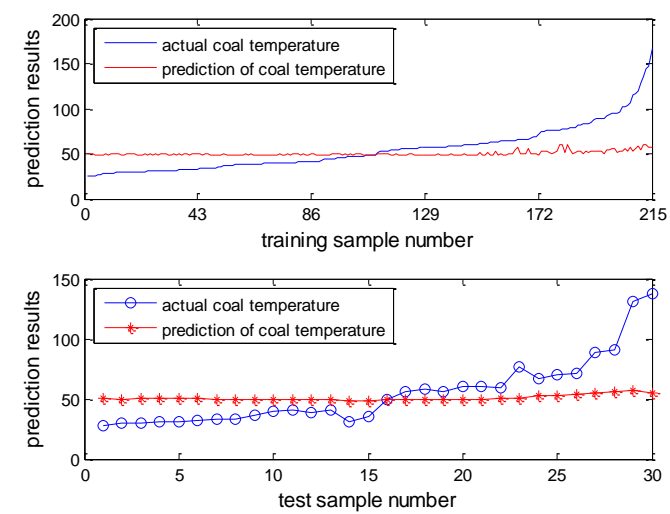

Fig. 6. The SVM regression model training and prediction results.
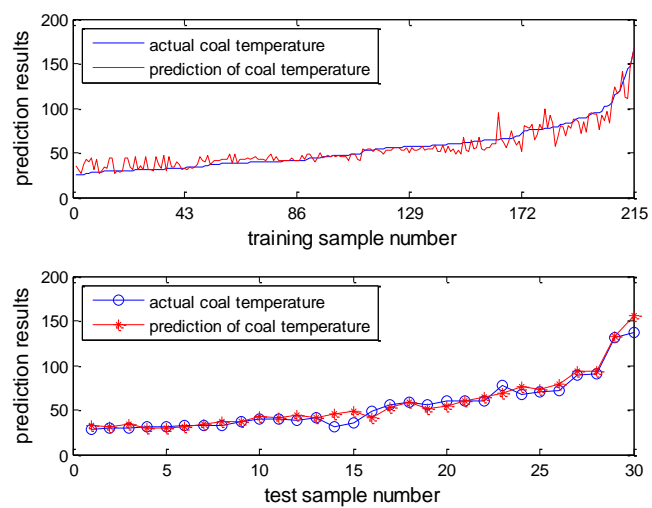

Fig. 7. PSO - SVM regression model training and prediction results.

\section{Decision Level Fusion Recognition}

According to the results of the data level and feature level, the prediction methods of decision level fusion recognition is as follows: in order to determine the danger grade of coal spontaneous combustion, it can be classified according to the classification results of PSO - SVM. In order to determine temperature, the test data can be classified by the least squares fitting temperature value and PSO - SVM regression. If temperature value fitted by the least squares and the PSO SVM regression results are not in the same level range, the classification prediction value of PSO - SVM can be determined the temperature value.

\section{CONCLUSIONS}

1) Coal spontaneous combustion process is divided into three stages: slow oxidation, accelerating oxidation and intense oxidation according to the development change rule of gas concentration and their ratio along with the coal temperature. The sensitivity indicators of quantitative identification for the degree of coal spontaneous combustion are determined, the critical temperature: $60 \sim 70{ }^{\circ} \mathrm{C}$, the pyrolysis temperature: $105 \sim$ $135^{\circ} \mathrm{C}$, the fission temperature: $135 \sim 145^{\circ} \mathrm{C}$.

2) The prediction method of fusion recognition for characteristic information of coal spontaneous combustion is put forward, and the anticipation mechanism is determined based on SVM algorithm and 
mathematical model.

3) Combined with the test data, data level adopts polynomial least squares fitting method, the correlation of the coal temperature and the indexes are established. According to the temperature of the gas concentration fitting out, it has carried on the quadratic fitting, then the quantitative relationship of the characteristic temperature of coal spontaneous combustion and the gas concentration of each index was determined.

4) In feature level, the prediction model of PSO - SVM fusion recognition is established, PSO is used to analyse the SVM parameters $\mathrm{C}$ and $\gamma$ search optimization, SVM and kernel function parameters optimization is implemented, the accuracy of classification prediction is approximate $100 \%$, the prediction accuracy of the danger degree of coal spontaneous combustion is improved.

5) In decision level, according to the results of the data level and feature level, the diagnosis and early warning criterion of coal spontaneous combustion is given, the classification anticipation criteria of the dangerous degree of coal spontaneous combustion is identified, the classification anticipation and effective decision-making of the dangerous degree of coal spontaneous combustion is achieved.

The error of fitting function is discussed in the future work. Furthermore, the mechanism of coal spontaneous combustion is revealed based on experiment.

\section{REFERENCES}

[1] Y. Tao, "Control mechanism and technology for integrated disaster of gas and coal spontaneous combustion in Gob," Ph.D. dissertation, University of Science and Technology of China, 2014.

[2] X. Wang, "Study on prevention and control technology of spontaneous combustion for large area goaf of Nantun CoalMine," M.S. thesis, Xi 'an University of Science and Technology, 2014.

[3] X.-K. Chen, "Study on early warning method for coal spontaneous combustion based on multi-information fusion," Ph.D. dissertation, Xi 'an University of Science and Technology, 2012.

[4] J.-F. Zhu et al., "The relationship between oxygen consumption rate and temperature during coal spontaneous combustion," Safety Science, vol. 50, no. 4, pp. 842-845, April 2012.

[5] L. Li et al., "Experimental study on temporal variation of high temperature region and index gas of coal spontaneous combustion," Journal of the China Coal Society, vol. 41, no. 2, pp. 444-450, February 1, 2016.

[6] Moni, Klouda, and Kudelova, "Research of possibilities of prognosis of brown coal spontaneous combustion sources genesis," International Multidisciplinary Scientific Geo Conference Surveying Geology and Mining Ecology Management, SGEM, vol. 3, no. 1, pp. 481-488, 2014

[7] L. Tana et al., "Mine hazards - assessing the hazard of coal spontaneous combustion," in Proc. 30th Annual International Pittsburgh Coal Conference 2013, vol. 2, pp. 1016-1045, 2013.
[8] R. Sara et al., "Multi-agent information fusion system to manage data from a WSN in a residential home," Information Fusion, vol. 23, pp. 43-57, May 2015.

[9] R. Caballero-Águila et al., "Fusion estimation using measured outputs with random parameter matrices subject to random delays and packet dropouts," Signal Processing, vol. 127, pp. 12-23, October 2016.

[10] C. Simone, "Equivalence of measurement space solution data fusion and complete fusion," Journal of Quantitative Spectroscopy and Radiative Transfer, vol. 182, pp. 71-74, October 1, 2016.

[11] S. Fayçal et al., "Bayesian estimation via extended and unscented Kalman particle filtering for non-linear stochastic systems," in Proc. 14th International Conference on Sciences and Techniques of Automatic Control and Computer Engineering, 2013, pp. 89-95, 2013.

[12] N. Harish et al., "Neural network modelling, simulation and prediction of innovation growth in United Arab Emirates (UAE)," Procedia Computer Science, vol. 36, pp. 269-275, 2014.

[13] B. Jing et al., "Parameter optimization and application of support vector machine based on parallel artificial fish swarm algorithm," Journal of Software, vol. 8, no. 3, pp. 673-679, 2013.

[14] X.-L. Wu et al., "Landslide susceptibility prediction using GIS and PSO-SVM," Geomatics and Information Science of Wuhan University, vol. 41, no. 5, pp. 665-671, May 1, 2016.

[15] P. J. G. Nieto et al., "Hybrid PSO-SVM-based method for prediction of the remaining useful life for aircraft engines and evaluation of its reliability," Reliability Engineering and System Safety, vol. 138, pp. 219-231, June 2015.

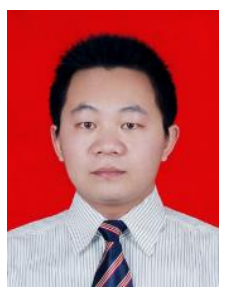

Wei-Feng Wang was born in June 1982 in Zhoukou city, Henan province. He is doctoral candidate in safety technology and engineering, Xi'an University of Science and Technology, Xi'an, Shaanxi, China. He mainly engaged in coal fire disaster prevention and control theory and technology research.

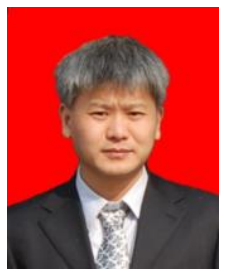

Jun Deng was born in June 1970 in Dazhu County, Sichuan province. He is executive vice president, Safety Science and Engineering State Key Disciplines Leader, Shaanxi Key Laboratory Director of Prevention and Control of Coal Fire, Xi'an University of Science and Technology, Xi'an, Shaanxi, China. He mainly engaged in coal fire disaster prevention and control theory and technology research.

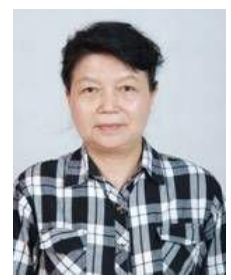

Yuan-Bin Hou was born in November 1953 in Xi'an, Shaanxi province. She is professor and the member of Education Committee of China Automation Association, the member of China Automation Association Electrical Specialized Committee, the executive director and Education Committee of Shaanxi Automation Association.

Nai-Guo Wang was born in October 1971 in Weifang City, Shandong province. He is senior engineer. He is the chief of the Xiezhuang Coal Mine of Shandong Energy Xinwen Mining Group Co., Ltd. He is mainly engaged in coal mine safety production technology and management. 